in vivo $34: 609-614(2020)$

doi:10.21873/invivo.11814

\title{
Evaluation of Goose-beak Bone Particles for Dentoalveolar Reconstruction in Dogs
}

\author{
SEOK JIN JANG ${ }^{1}$ and SEOK HWA CHOI ${ }^{2}$ \\ ${ }^{1}$ Onnuri Animal Medical Center, Cheongju, Republic of Korea; \\ ${ }^{2}$ Department of Veterinary Surgery, College of Veterinary Medicine, \\ Chungbuk National University, Cheongju, Republic of Korea
}

\begin{abstract}
Background/Aim. Tooth extraction is a common procedure in dental clinics. Tooth extraction can destroy gingiva, alveolar bone, periodontal ligaments and cement. If dental sockets are left as extracted, it will result in loss of teeth, as well as voice and aesthetic problems. A natural hydroxyapatite (HA) bioceramic bone graft substitute developed from goose-beak bone particles (GBPs) was used for dentoalveolar reconstruction in a canine model. Materials and Methods: Four adult (18-22 months old) male beagle dogs weighing 8.2-9.6 kg were included in the study. Eight alveolar extraction sockets in the four dogs were divided randomly into two groups and a split-mouth design was established; control group, socket filled with commercial synthetic HA; tested group, socket filled with granulated GBP. Results: Micro-CT analysis and hematoxylin and eosin and Masson's trichrome staining of non-decalcified sections were undertaken. Examination revealed that dentoalveolar reconstruction was initiated from the periphery of the host bone, and newly formed bone was well integrated with the $G B P$. Bone apposition was observed at the edge of the host bone-GBP interface. Conclusion: A natural ceramic powder obtained from GBP is suitable for use in dentoalveolar reconstruction in dogs.
\end{abstract}

Jawbone diseases are often the result of accidents, surgical removal of malignant neoplasms, congenital abnormalities, periodontitis, tooth abscess, or extraction (1). For the repair of bone defects, natural bone grafts or artificial synthetic

This article is freely accessible online.

Correspondence to: Seok Hwa Choi, DVM, Ph.D., Department of Veterinary Surgery, College of Veterinary Medicine, Chungbuk National University, Chungdae-ro 1, Seowon-Gu, Cheongju, Chungbuk, 28644, Republic of Korea. Tel: +82 432613144, Fax: +82 432613224, e-mail: shchoi@cbnu.ac.kr

Key Words: Goose-beak particles, dentoalveolar reconstruction, dog. bone grafts have been used. An ideal graft material should be biocompatible, safe, non-allergenic, non-toxic, and with no potential for disease transmission. Moreover, it should be sufficiently strong to be maintained in the graft space and exhibit an acceptable rate of degradation (2). Synthetic hydroxyapatite (HA) is commonly used as a bone graft substitute due to its inherent bioactive properties that support osteoconduction when used for bone regeneration. However, HA-based biomaterials have a weakness as they exhibit slow degradation and absorption in vivo. According to previous studies, the porous scaffold form of calcium phosphate ceramics such as HA can improve bone regenerative effects within canine alveolar sockets (3). However, this form of material needs an appropriate size and three-dimensional (3D) porosity in order to demonstrate its intrinsic osteoconductivity and osteogenic potential within limited and exiguous sites such as dental sockets. HA scaffold material with an interconnected pore diameter of $300 \mu \mathrm{m}$ has shown the most appropriate osteoconduction capacity (4).

Domestic geese are mainly used for meat, egg, and feather production, and their oval-shaped heads are usually discarded during processing. Goose beaks are composed of keratin, and comprise two major bones on each side of the upper and lower bill. Geese have a relatively low probability of harboring a zoonotic disease, and goose-beak-derived xenografts may be a suitable xenograft material for bone grafting. Goose-beak bone is a natural bioderived HA with some important advantages over other HA products: for example, as a discarded byproduct of goose farming it is available at a very low material cost, and it can be processed easily and quickly. As an alternative material to autogenous bone, goose-beak bone particles (GBPs) have attracted attention as a bone substitute due to their biological properties (5). The most widely used calcium phosphate ceramic is HA whose molar ratio of calcium to phosphorus ranges from 1.2 to 2 , which is very similar to that of human bone. The atomic $\mathrm{Ca} / \mathrm{P}$ ratio of $\mathrm{GBP}$ is reported to be 1.63 , and previous examination has not revealed any distinguishable crystalline impurities (5). 
Quantitative radiographic evaluation using X-rays has been performed to determine the structural and mechanical properties of bone indirectly. In particular, quantitative computed radiography (CT), and peripheral CT have been used in human medicine for monitoring and treatment of metabolic diseases, skeletal status, and osteoporosis (6). In recent years, micro-CT has been used as a means to provide more detailed information about bone mass and microstructure and as a way to obtain more accurate measurements. In addition, it has been used to assess bone metabolism in an animal model (7). It has been assumed that bone formation and mineralization will affect bone strength, and mechanical stability has been shown to depend on the amount and density of bone (8). Bone regeneration abilities of bone substitutes have been compared in a bone metabolic disorder model by assessing bone mineral density (BMD), percent bone volume, and other bone structural parameters. In this study, GBPs were inserted in mandibular dental sockets in beagle dogs and BMD changes were assessed by using CT while bone parameter values were obtained by micro-CT at different times post-insertion. After dental extraction, alveolar ridge maintenance is a requisite for successful healing and for reducing bone dimensional changes during healing. The purpose of this study was to evaluate and compare dentoalveolar reconstruction in canine dental sockets when using commercial synthetic HA or bioceramic GBP.

\section{Materials and Methods}

Goose-beak-derived bone particles. In order to collect suitable goosebeak bone tissue material from harvested domestic geese (Emden breed, males, 17-week-old), all soft tissues were removed from the goose beaks, and the associated bone was surgically extracted. The obtained bone samples were thoroughly washed to remove macroscopically adhered impurities and then boiled in distilled water for $8 \mathrm{~h}$. The boiled samples were then deproteinized by treating with hydrogen dioxide for $8 \mathrm{~h}$ (9) followed by drying for $48 \mathrm{~h}$ in an oven at $60^{\circ} \mathrm{C}$. The prepared goose-beak bone samples were then cut into pieces smaller than $2 \mathrm{~cm}^{3}$, followed by rinsing with distilled water to remove any remaining blood or hydrogen dioxide. Through the above processes, all organic matrices and potential disease-causing agents were removed from the samples. For use, the sintered goose-beak bone pieces were milled to obtain 500-700 $\mu \mathrm{m}$ particles. To remove any moisture residue totally, the milled bone particles were freezedried and then maintained at room temperature (5).

In vivo study. Four adult (18-22 months old) male beagle dogs, weighing between 8.2 and $9.6 \mathrm{~kg}$, were included in this study. The animal experiment protocol was approved by the Institutional Animal Care and Use Committee of Chungbuk National University (Approval CA-17-22). All dogs were kept in individual cages throughout the experimental period. Water and food were supplied ad libitum during the experimental period.

Surgical procedures were performed under general anesthesia and sterile conditions. The left and right mandibular first molars were
Table I. BMD and bone volume 8 weeks after insertion of goose-beak bone particles in canine alveolar sockets.

\begin{tabular}{lll}
\hline Group & BMD $\left(\mathrm{g} / \mathrm{cm}^{3}\right)$ & BV/TV $(\%)$ \\
\hline Control & $0.57 \pm 0.15$ & $61.72 \pm 6.54$ \\
Tested & $0.24 \pm 0.10^{*}$ & $19.03 \pm 10.93^{*}$ \\
\hline
\end{tabular}

Data are expressed as mean $\pm S D(n=4) . *$ Significant difference from control group at $p<0.05$. BMD: Bone mineral density; $\mathrm{BV}$ : bone volume; TV: tissue volume.

extracted without injury by using the closed extraction technique. Closure of the graft site was accomplished with 4-0 single interrupted absorbable sutures (Surgifit, Ailee Co., Busan, Republic of Korea) and using the gingival flap technique. Enough tissue was elevated for the gingival flap to be placed over the socket without spontaneous retraction. During the experimental period, treated dogs were fed commercial dog feed soaked in water in order to minimize damage to the surgical area. Antibiotics and analgesics were administered in the postoperative period.

The eight dental extraction sites were divided randomly using a split-mouth design to establish two study groups: control group: four dental sockets filled with commercial synthetic HA $\left[\mathrm{Ca}_{5}(\mathrm{OH})\left(\mathrm{PO}_{4}\right)_{3},<200 \mathrm{~nm}\right.$ particle size, Sigma-Aldrich Co., St Louis, MO, USA]; test group: four dental sockets filled with granulated GBP.

Dental computed tomography imaging. At 8 weeks after dental surgery, dental CT scans of the head were obtained under general anesthesia in the same manner as described above in the in vivo study section. Each dog was positioned in ventrodorsal recumbency on the cradle of a CT scanner, and its head position was adjusted for symmetry. High-resolution transverse imaging of the mandible of each dog was obtained with a conventional CT scanner (CTHiSpeed Advantage ${ }^{\mathrm{TM}}$, General Electric Co., TX, USA). Images were taken contiguously at $1 \mathrm{~mm}$ intervals with a resolution close to $0.25 \mathrm{~mm} /$ pixel at $130 \mathrm{kVp}$ and $20 \mathrm{mAs}$ using a standard dental $\mathrm{CT}$ investigation protocol. The thin transverse image data obtained from the mandible were reformatted using specialized software (10DR ${ }^{\circledR}$ Implant Service Co., Seoul, Republic of Korea) to automatically produce cross-sectional images, curved linear panoramic images, and 3D reconstruction images every 1 to $2 \mathrm{~mm}$ around the dental arch. The CT images were reconstructed in order to define the bone contours.

Preparation of specimens for fluorescence microscopy. To label the mineralized tissue and assess the time course of new bone formation and mineralization, a polychrome sequential fluorescent labeling method was used. Oxytetracycline $(25 \mathrm{mg} / \mathrm{kg}$; Green Cross Co., Yongin, Gyeonggi, Republic of Korea) and calcein $(20 \mathrm{mg} / \mathrm{kg}$, Sigma-Aldrich Co.) were injected intravenously at four and six weeks after HA or GBP insertion. Alizarin red s $(30 \mathrm{mg} / \mathrm{kg}$, SigmaAldrich Co.) was intraperitoneally injected at 8 weeks after HA or GBP insertion. Samples obtained from the sacrificed alveolar sockets in each beagle were fixed in formalin and dehydrated in a graded series of ethanol, and then immersed in acrylic resin using Technovit 7200VLC (Technovit; Hatfield, PA, USA) before undergoing light catalysis for $24 \mathrm{~h}$. A macro-cutting and grinding 

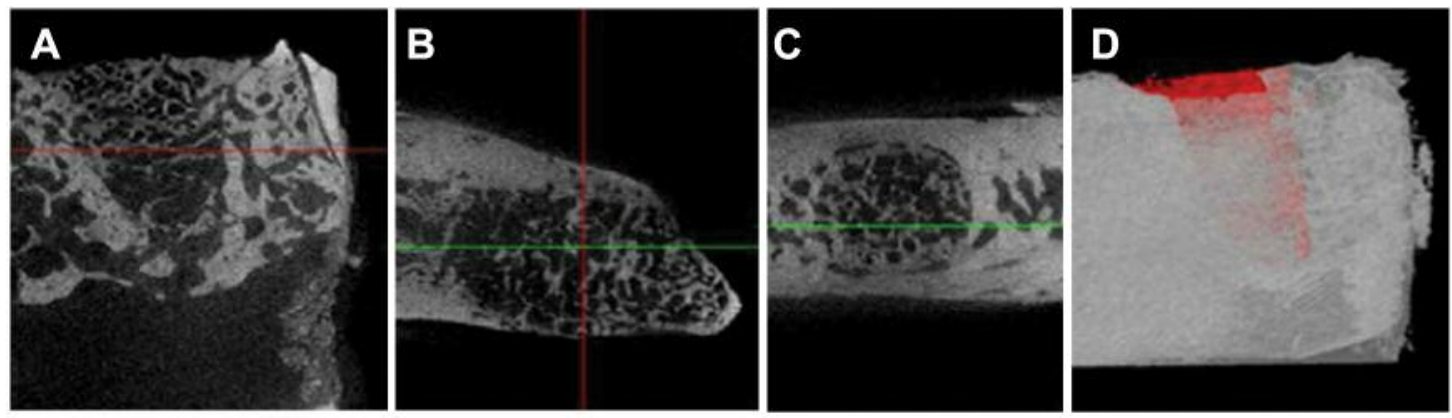

Figure 1. Micro-CT images at 8 weeks post-insertion of goose-beak particles in canine dental sockets. Transaxial (A), sagittal (B), coronal (C), and $3 D$ images $(D)$.
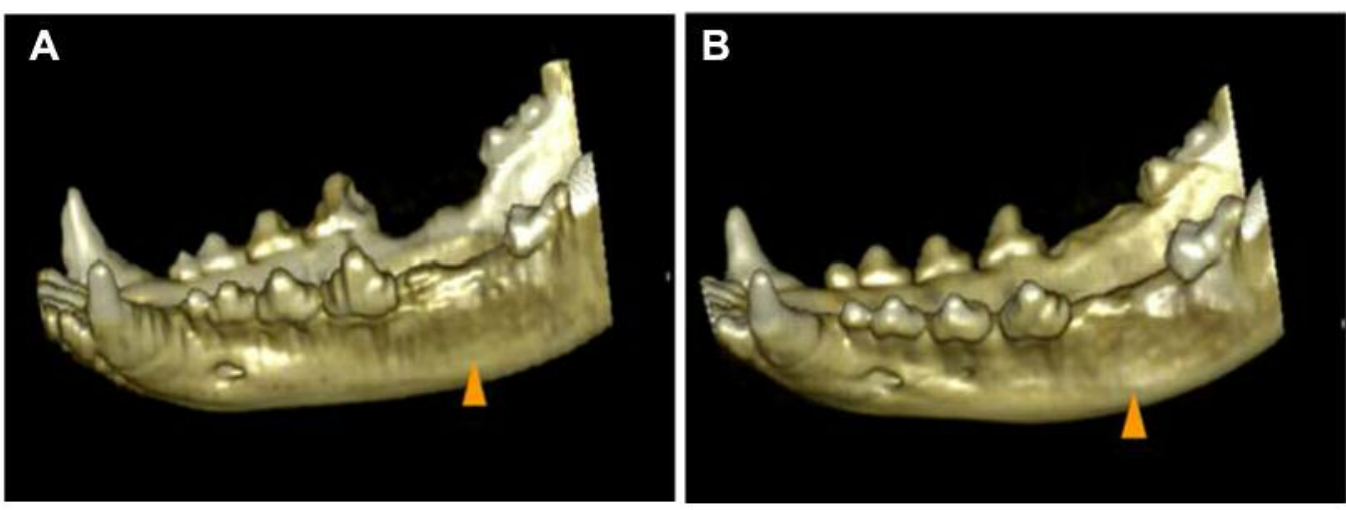

Figure 2. Three-dimensional CT images at 8 weeks post-insertion of synthetic hydroxyapatite (A) and goose-beak bone particles (B) into canine dental sockets. The head of arrow indicates insertion site.

system (Exakt 310CP series; Exakt Apparatebau, Norderstedt, Germany) was used to obtain non-decalcified cut and ground sections. A final section thickness of approximately $50 \mu \mathrm{m}$ was used for fluorescence microscopy. Fluorescent labeling of new bone formation was evaluated via confocal laser scanning microscopy. Active bone formation was evaluated by assessing the presence or absence, intensity, and width of the fluorochrome markers.

Histological evaluation. After scanning to obtain micro-CT images, mandibular tissues around the defect sockets were harvested and fixed for $24 \mathrm{~h}$ in a $10 \%$ formalin solution at room temperature. Subsequently, the samples were dehydrated in an ascending series of alcohol rinses. Dehydrated samples were then embedded in acrylic resin (Technovit 7200 VLC; Kulzer \& Co GmbH, Friedrichdorf, Germany) and serially cut into $20 \mu \mathrm{m}$ thick sections. Section specimens were then mounted on glass slides and stained with hematoxylin and eosin (H\&E) and Masson's trichrome (MT). Microscopy images were obtained with a light microscope.

Statistical analysis. Statistical analyses were performed using SPSS software version 19.0.1.1. (IBM SPSS Statistics for Windows, Version 19.0; IBM Corp., Armonk, NY, USA). Data are presented as mean \pm standard deviation $(\mathrm{SD})$ values. Normality and homogeneity of the data were confirmed before performing an analysis of variance (ANOVA). Differences among the experimental groups were assessed by performing one-way ANOVA followed by Duncan's multiple range tests. Null hypotheses of no significant difference were rejected if the obtained $p$-values were less than 0.05 .

\section{Results}

Findings of micro-CT. The BMD values and percent bone volumes over time in the two experimental groups are presented in Table I. The results show a general increase in BMD for both groups over the 8-week experimental period, but at 8 weeks post-insertion, BMD values and percent bone volumes were significantly different between the control HA and test GBP groups $(p<0.05$, Table I).

The $3 \mathrm{D}$ reconstructions of the bone grafts at 8 weeks postinsertion, which were obtained by examining tomographic sections, showed dentoalveolar reconstruction following alveolar ridge augmentation in the GBP insertion sites. Bone height apparently occurred in the GBP insertion sites and the ridge height response demonstrated that bone formation was 


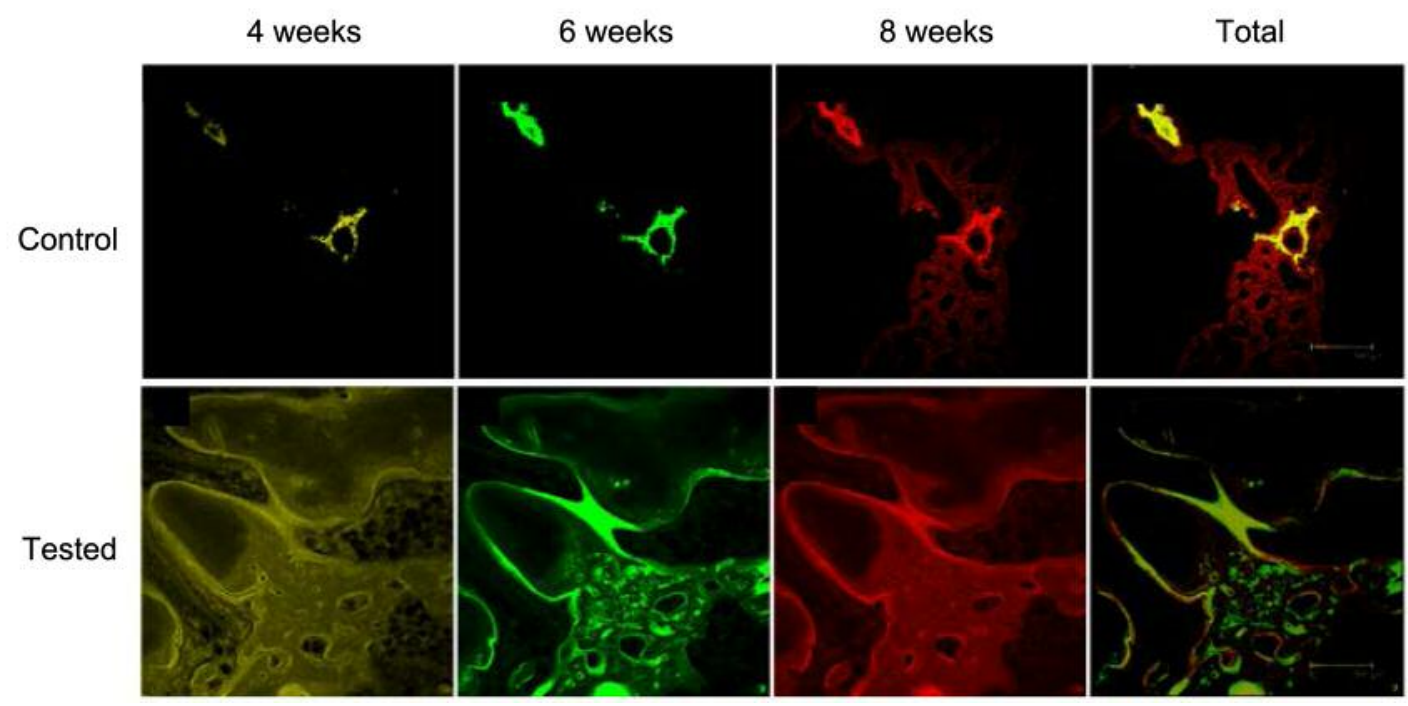

Figure 3. Confocal LASER microscope images of synthetic hydroxyapatites (control) and goose-beak particles insertion (tested). New bone formation and mineralization was determined by tetracycline (yellow), calcein (green), and alizarin red $S$ (red) fluorescent quantification, which represented the mineralization level at 4, 6, and 8 weeks post-insertion.

equivalent to forming a full filling of the extraction socket defect (Figures 1 and 2).

Histological findings. Newly formed bone in the GBP insertion region was observed in the non-decalcified specimens stained with oxytetracycline, calcein, and alizarin red s. When observed under a confocal microscope, yellow (oxytetracycline), green (calcein), and red (alizarin red S) staining of specimen sections revealed the presence of new bone formation. No complications, such as infection and inflammation, were detected among the examined specimens. Dentoalveolar reconstruction was observed from native basal bone (peripheral region) to the center of the alveolar socket. New bone was observed around host bone. Dentoalveolar reconstruction originated at the periphery of the host bone, and newly formed bone was well integrated with the GBP. Bone apposition was observed at the edge of the interface between the host bone and the GBP (Figure 3).

\section{Discussion}

Tooth extraction is a common procedure in dental clinics. The normal healing response to this treatment results in significant bone loss and collapse of the surrounding gums. In addition to the effects of normal healing, a significant proportion of the extraction sites suffer from post-extraction complications. An important healing requirement after tooth extraction is maintenance of the alveolar ridge against bone dimensional changes. Implanting a bone substitute into an extraction socket is reported to have a positive effect on bone healing and to prevent atrophy of the surrounding alveolar bone (10).

HA is one of the most widely used calcium phosphate biomaterials in bone research and related clinical fields. Recently, researchers have attempted to extract HA from natural sources such as corals, seashells, egg shells, cuttlefish shells, natural gypsum, and naturally occurring calcite (11). Biomedical ceramics, because of their superior mechanical properties and biocompatibilities compared to those of polymer materials, are widely used when treating or repairing bone loss or damage in various medical fields, such as dental surgery, orthopedics, and plastic surgery $(11,12)$. HA is a bioactive substance with a composition and structure similar to that of natural bone (12) and, as it induces alveolar growth, can be used to treat alveolar bone defects.

Many xenografts can be produced at relatively affordable prices. However, a disadvantage of xenografts is that their processing procedures can influence their physicochemical properties. The most widely used calcium phosphate ceramic xenograft is $\mathrm{HA}$ whose molar $\mathrm{Ca} / \mathrm{P}$ ratio ranges from 1.2 to almost 2, which is very similar to that of human bone. The GBP xenograft material in this study had a $\mathrm{Ca} / \mathrm{P}$ ratio of 1.63. Moreover, a previous study did not reveal any distinguishable crystalline impurities in GBP (5).

Clinical, radiological, and micro-CT parameters (e.g., probing depth, clinical attachment level, intrabone defect depth, and percentage of defect filling) are generally used to assess that state of dentoalveolar reconstruction (7), and healing progress is primarily assessed on the basis of radiographic changes in the grafted area. Although two- 
dimensional assessment of dentoalveolar socket remodeling at the insertion site of bone graft substitutes can be evaluated by conventional radiographs, such conventional visual analysis is not sufficiently precise to detect subtle changes in bone density (13). CT appears to be the best radiological method for morphological and qualitative analysis of bone, and an additional advantage of measurement via CT is that bone density can also be calculated (14). In our gross evaluation of CT-based 3D reconstruction of canine mandibles after 8 weeks of HA or GBP insertion, the areas inserted with GBP exhibited a greater amount of bone augmentation than that in areas with HA insertion and the GBP insertions produced excellent thickness on the buccal aspect of the alveolar ridge in dogs. These results showed that the natural bioceramic GBP can act as an osteoconductive graft material in dentoalveolar reconstruction of canine alveolar sockets.

The volume and morphology of new bone formed in an alveolar socket can be assessed by examining 3D images that allow discrimination of the area of the socket from the surrounding normal alveolar bone $(7,15)$. In our study, new bone was observed at dentoalveolar sockets after GBP insertion on micro-CT 3D reconstructed images. New bone formation at the interface between the insertion graft material and the host bone was obvious in the GBP-treated group, with the graft biomaterial appearing radiopaque due to its structural properties. Moreover, there was no peripheral alveolar bone regression or bone resorption observed in the areas adjacent to the GBPs inserts.

Micro-CT results showed that the dentoalveolar reconstruction effects were greater in the GBP group than in the HA group. The GBP-treated group showed a high level of new bone formation originating from the extraction site margin and a good level of dentoalveolar reconstruction. These results further indicate that the GBP bioceramic provided better osteoconduction and osteoinduction than the commercially available synthetic HA. In addition, the results showed that GBP can be used as an autograft substitute in dogs. The crystallized GBPs used in this study had a high surface area, large pore size, and high pore volume as a result of the heating process; these structural properties are very important for osteoblast and bone regeneration (5).

The dentoalveolar reconstruction process was confirmed by histopathological examination. When dentoalveolar reconstruction started, osteoblasts formed, and the osteoblasts subsequently became osteocytes and osteoclasts. The bioceramic GBPs produced better dentoalveolar reconstructive effects than those from the commercially available synthetic HA. In the GBP group, new bone formation began from the dental sockets, and the lacunae of GBPs became filled with new osteocytes. These results are consistent with a previous report indicating that a bioceramic HA obtained from GBPs is suitable for use in bone regeneration applications (16). In the current study, osteoblastic cells were observed on the GBP surface, indicating the initiation of partially mineralized osteoid formation. That osteoid formation then matured into fully mineralized bone, resulting in firm bonding of the bone to GBP. In contrast, HA insertion into dental defects results in the formation of small apatite crystals centered in aggregates between relatively large HA crystals. This is similar to those observed in adjacent dental defects which can produce similar diffraction patterns (17).

A periodontal regeneration study showed that HA has an excellent regenerative effect compared to that achieved from open flap debridement (18). Some modified HA materials have been shown to improve protein adsorption (19), and the use of HA has gained special interest due to its compositional similarity to natural bone. GBPs subjected to heat treatment, sintering, develop the particles to form a porous, solid material. Mammalian bones are hard, but goose bones are porous and soft, making them easy to process; and by modifying the GBP processing method, particles of various sizes can be produced. These advantages indicate that GBPs are suitable for use in dentoalveolar reconstruction and can be used in various shapes and forms. In conclusion, a bioceramic powder obtained from domestic goose beak bones is suitable for dentoalveolar reconstruction of canine dental extract sockets.

\section{Conflicts of Interest}

The Authors declare that they have no competing interests regarding this study.

\section{Authors' Contributions}

This study was designed by Jang and Choi. Jang and Choi analyzed the CT image and data collection. Both Authors read and approved the final manuscript.

\section{Acknowledgements}

This research was supported by the Basic Science Research Program provided through the National Research Foundation of Korea (NRF) and funded by the Ministry of Education (2018R1D1A1A09083556).

\section{References}

1 Buser D, Dula K, Hess D, Hirt HP and Belser UC: Localized ridge augmentation with autografts and barrier membranes. Periodontol 1999: 151-163, 2000. PMID: 10321222. DOI: 10.1111/j.1600-0757.1999.tb00153.x

2 Darby I: Periodontal materials. Aust Dent J 56(S1): 107-118, 2011. PMID: 21564121. DOI: 10.1111/j.1834-7819.2010.01301.x

3 Jang SJ, Kim SE, Han TS, Son JS, Kang SS and Choi SH: Bone regeneration of hydroxyapatite with granular form or porous scaffold in canine alveolar sockets. In Vivo 31(3): 335341, 2017. PMID: 28438860. DOI: 10.21873 /invivo.11064 
4 Chang BS, Lee CK, Hong KS, Youn HJ, Ryu HS, Chung SS and Park KW: Osteoconduction at porous hydroxyapatite with various pore configurations. Biomaterials 21: 1291-1298, 2000. PMID: 10811311. DOI: 10.1016/S0142-9612(00)00030-2

5 Kim JM and Choi SH: Osteogenic evaluation of goose-beak bones according to processing temperature. In Vivo 33: 419-424, 2019. PMID: 30804120. DOI: 10.21873/invivo.11489

6 Ebbesen EN, Thomsen JS, Beck-Nielsen H, Nepper-Rasmussen HJ and Mosekilde L: Lumbar vertebral body compressive strength evaluated by dual-energy X-ray absorptiometry, quantitative computed tomography, and ashing. Bone 25: 713-724, 1999 PMID: 10593417. DOI: 10.1016/s8756-3282(99)00216-1

7 Schouten C, Meijer GJ, van den Beucken JJ, Spauwen PH and Jansen JA: The quantitative assessment of periimplant bone responses using histomorphometry and microcomputed tomography. Biomaterials 30: 4539-4549, 2009. PMID: 19500840. DOI: 10.1016/j.biomaterials.2009.05.017

8 Faulkner KG, Gluer CC, Majumdar S, Lang P, Engelke K and Genant HK: Noninvasive measurements of bone mass, structure, and strength: current methods and experimental techniques. AJR Am J Roentgenol 157: 1229-1237, 1991. PMID: 1950872. DOI: 10.2214/ajr.157.6.1950872

9 Kim JH, Park MH, Jang SJ, Son SJ, Lee JY, Son JS, Kim SE, Kang SS and Choi SH: Effect of hydrogen dioxide treatment on the osteogenic potential of duck-beak bone-derived natural bioceramic microparticles. In Vivo 31: 373-379, 2017. PMID: 28438865. DOI: 10.21873/invivo.11069

10 Rothamel D, Schwarz F, Herten M, Engelhardt E, Donath K, Kuehn $\mathrm{P}$ and Becker J: Dimensional ridge alterations following socket preservation using a nanocrystalline hydroxyapatite paste: a histomorphometrical study in dogs. Int J Oral Maxillofac Surg 37: 741-747, 2008. PMID: 18554868. DOI: 10.1016/j.jjom.2008.04.017

11 Son JS, Jang SH, Kwon TY, Kim KH, Kang SS and Choi SH: Preliminary evaluation of bone graft substitute produced by bone of duck beak. Materials Lett 121: 181-184, 2014. DOI: 10.1016/j.matlet.2014.01.141

12 Son JS, Appleford M, Ong JL, Wenke JC, Kim JM, Choi SH and Oh DS: Porous hydroxyapatite scaffold with three-dimensional localized drug delivery system using biodegradable microspheres. J Control Release 153: 133-140, 2011. PMID: 21420453. DOI: $10.1016 /$ j.jconrel.2011.03.010
$13 \mathrm{Kim}$ M, Kim JH, Lee JY, Cho K, Kang SS, Kim G, Lee MJ and Choi SH: Effect of bone mineral with or without collagen membrane in ridge dehiscence defects following premolar extraction. In Vivo 22: 231-236, 2008. PMID: 18468408.

14 Brett AD and Brown JK: Quantitative computed tomography and opportunistic bone density screening by dual use of computed omography scans. J Orthop Translat 3: 178-184, 2015. PMID: 30035056.

15 Cho HJ, Jeon JY, Ahn SJ, Lee SW, Chung JR, Park CJ and Hwang KG: The preliminary study for three-dimensional alveolar bone morphologic characteristics for alveolar bone restoration. Maxillofac Plast Reconstr Surg 41: 33, 2019. PMID: 31531306.

16 Kim JM, Han TS, Kim MH, Oh DS, Kang SS, Kim G, Kwon TY, Kim KH, Lee KB, Son JS and Choi SH: Osteogenic evaluation of calcium phosphate scaffold with drug-loaded poly (lactic-co-glycolic acid) microspheres in beagle dogs. Tissue Eng Regen Med 9: 175-183, 2012. DOI: 10.1007/s13770-012-01755

17 Palmer LC, Newcomb CJ, Kaltz SR, Spoerke ED and Stupp SI: Biomimetic systems for hydroxyapatite mineralization inspired by bone and enamel. Chem Rev 108: 4754-4783, 2008. PMID: 19006400.

18 Pradeep AR, Rao AS, Agarwal E, Bajaj P, Kumari M and Naik SB: Comparative evaluation of autologous platelet-rich fibrin and platelet-rich plasma in the treatment of 3-wall intrabony defects in chronic periodontitis: a randomized controlled clinical trial. J Periodontol 83: 1499-1507, 2012. PMID: 22348695. DOI: 10.1902/jop.2012.110705

19 Wei G and Ma PX: Structure and properties of nanohydroxyapatite/polymer composite scaffolds for bone tissue engineering. Biomaterials 25: 4749-4757, 2004. PMID: 15120521. DOI: 10.1016/biomaterials.2003.12.005
Received October 22, 2019

Revised November 13, 2019 Accepted November 15, 2019 